\title{
Aspectos morfológicos de biópsias musculares em equinos com miopatia sob forma de surto ${ }^{1}$
}

\author{
Rogério M. Amorim ${ }^{2 *}$, Adriana S. Rino ${ }^{2}$, Maeli Dal-Pai-Silva ${ }^{3}$, Alexandre S. Borges ${ }^{2}$, \\ José Paes Oliveira Filho ${ }^{2}$, Natália P.P. Freitas ${ }^{2}$, Leandro Maia ${ }^{2}$ e Luis A.L.Rezende ${ }^{4}$
}

\begin{abstract}
Amorim R.M., Rino A.S., Dal-Pai M., Borges A.S. Oliveira Filho J.P., Freitas N.P.P., Maia L. \& Rezende L.A.L. 2011. [Morphological features of muscle biopsies from horses of a myopathy outbreak.] Aspectos morfológicos de biópsias musculares em equinos com miopatia em forma de surto. Pesquisa Veterinária Brasileira 31(7):579-585. Departamento de Clínica Veterinária, Faculdade de Medicina Veterinária e Zootecnia, Universidade Estadual Paulista, Campus de Botucatu, Distrito de Rubião Júnior s/n, Botucatu, SP 18618-970, Brazil. Email: rmamorim@fmvz.unesp.br

Equine myopathies are classified according clinic, morphologic and molecular features in three groups: Non-exertional, exertional, and abnormal muscle membrane conduction. In spite the advances in diagnostic, the literature has reported outbreaks of equine myopathy without clear etiology in several countries. The aim of this study was to describe the histological and histochemical findings of muscle biopsies in an outbreak of equine myopathy. Seven 18 to 24month-old Quarter horses showed clinical signs of myopathy. Five horses presented mild clinical signs and two horses had severe clinical signs with recumbency. Muscle biopsies were obtained from gluteal medium muscle by percutaneous technique with Bergstrom needle in all affected horses. Muscle samples were processed by histological (HE, modified gomori-trichrome) and histochemical (PAS, NADH, ATPase) technics. In animals with mild clinical signs, ragged red fibers type I and IIA, related to the oxidative metabolism dysfunction of mitochondria, was the main abnormality found. Muscle fiber atrophy and presence of subsarcolemmal aggregates in type I and IIA muscle fibers were also observed. More severe affected horses presented inflammatory infiltrate, proliferation of collagen, phagocytosis, necrosis and calcification. Based on the morphological findings, vitamin E therapy response associated with the low mortality when compared with atypical myopathy reports, We concluded that this outbreak was triggering for mitochondrial lesions, characterized by ragged red muscle fibers, probably due a breakdown homeostasis in vitamin $\mathrm{E}$ and Se, being compatible with nutritional myopathy diagnosis.
\end{abstract}

INDEX TERMS: White muscle disease, nutritional myopathy, atypical myopathy, ragged red fibers.

RESUMO.- As miopatias em equinos são classificadas de acordo com aspectos clínicos, morfológicos e moleculares, em três grandes grupos: não associadas ao exercício, associadas ao exercício e devido alteração da condução elétrica do sarcolema. Apesar dos avanços no diagnóstico, a literatura ainda relata

\footnotetext{
${ }^{1}$ Recebido em 22 de outubro de 2010.

Aceito para publicação em 17 de março de 2011.

${ }^{2}$ Departamento de Clínica Veterinária, Faculdade de Medicina Veterinária e Zootecnia, Universidade Estadual Paulista (Unesp), Campus de Botucatu, Distrito de Rubião Júnior s/n, Botucatu, SP 18618-970, Brasil. *Autor para correspondência: rmamorim@fmvz.unesp.br

${ }^{3}$ Departamento de Morfologia do Instituto de Biociências, Unesp, Campus de Botucatu, Distrito de Rubião Júnior s/n, Botucatu, SP.

${ }^{4}$ Departamento de Neurologia e Psiquiatria, Unesp, Campus de Botucatu, Distrito de Rubião Júnior s/n, Botucatu, SP.
}

surtos de miopatia em equinos sem etiologia esclarecida em diversos países. 0 objetivo desse estudo foi descrever as alterações histológicas e histoquímicas de biópsias musculares de equinos acometidos por miopatia. Sete equinos da raça Quarto de Milha, com 18-24 meses de idade, apresentaram sinais clínicos de miopatia. Dentre esses animais, cinco apresentaram sinais subagudos leves a moderados e dois apresentaram sinais hiperagudos severos e decúbito lateral. Foram realizadas biópsias musculares utilizando a técnica percutânea, por agulha tipo Bergström, no músculo glúteo médio em todos os animais acometidos. As amostras foram processadas por meio de técnicas histológicas (HE, Tricrômio de Gomori modificado) e histoquímicas (PAS, NADH, ATPase). Nos quadros clínicos mais leves, a principal alteração encontrada foi a presença de fibras vermelhas rajadas do tipo I e 
IIA, que estão associadas às alterações do metabolismo oxidativo e das funções mitocondriais, como ocorrem nas miopatias mitocondriais. Também foram observadas fibras atróficas do tipo I e IIA, além da presença de agregados subsarcolemais. Nos quadros mais severos o tecido muscular apresentou infiltrado inflamatório, aumento de colágeno, fagocitose, necrose, calcificação e regeneração muscular. Diante dos achados morfológicos, da resposta à terapia com vitamina $\mathrm{E}$ e Se e da baixa mortalidade quando comparado aos relatos de miopatia atípica, conclui-se que esse surto foi desencadeado por lesões mitocondriais, caracterizadas pelas fibras musculares vermelhas rajadas, possivelmente devido uma quebra da homeostase de vitamina E e Se, sendo compatível com o diagnóstico de miopatia nutricional.

TERMOS DE INDEXAÇÃO: Doença do músculo branco, miopatia nutricional, miopatia atípica, fibras vermelhas rajadas.

\section{INTRODUÇÃO}

As miopatias são relativamente comuns na espécie equina e no passado receberam nomes como: "mal da segunda-feira", azotúria, mioglobinúria paralítica, rabdomiólise e tying up. Atualmente sabe-se que as mesmas manifestações clínicas são comuns a diferentes etiologias (Valberg et al. 1999, Aleman 2008), sendo classificadas em três grandes grupos: não associadas ao exercício, associadas ao exercício e devido alteração da condução elétrica do sarcolema (Aleman 2008).

0 grupo das miopatias não associadas ao exercício inclui as de causa nutricional (deficiência de vitamina E e Se) (Lofestd 1997), metabólica (deficiência da enzima I de ramificação do glicogênio e por acúmulo de polissacarídeo) (Valberg et al. 1992, Ward et al. 2004), tóxica (Martin et al. 1981, Bezerra et al. 2000, Cassart et al. 2007), traumática, inflamatória (bacterianas, virais, parasitárias e imunomediadas) (Peek et al. 2003, Sponseller et al. 2005, Lewis et al. 2007, Aleman 2008), associada a anestesia (miopatia isquêmica e hipertermia maligna) (Aleman 2008), induzida por esteróides, neoplásicas e síndromes paraneoplásicas (Aleman 2008).

Miodegeneração nutricional, também conhecida como distrofia muscular nutricional, miodegeneração distrófica, miodistrofia nutricional ou doença do músculo branco, é uma afecção hiperaguda, aguda ou subaguda da musculatura estriada cardíaca e/ou esquelética causada pela deficiência de selênio (Se) e ocasionalmente vitamina $\mathrm{E}$ (tocoferol) (Higuchi et al. 1989).

A miopatia nutricional ocorre mais comumente em equinos criados sob manejo extensivo estando assim, sujeitos à deficiência de vitamina E e Selênio. Plantas de rápido crescimento e/ou cultivadas em solos repetidamente adubados com fertilizantes contendo enxofre, ácidos, com altas concentrações de ferro tendem a ter baixas concentrações de Se. Assim como rações peletizadas podem ser deficientes nesses dois antioxidantes (Vitamina E e Se) (Lofstedt 1997, Aleman 2008).

O Se é um componente essencial de cinco selenoproteínas, incluindo enzimas glutationa peroxidase (Burk \& Hill 1993). A vitamina $\mathrm{E}$ e a glutationa peroxidase agem como antioxidantes biológicos, por protegerem as organelas lipídicas e as membranas celulares dos danos peroxidativos causados pelas espécies reativas do oxigênio (ERO). As ERO são estrutu- ras químicas que podem se formar por reações endógenas, geralmente oxidativas, durante processos metabólicos normais da célula (Combs \& Combs 1986).

A vitamina E, uma molécula lipofílica, age como antioxidante na membrana celular e remove as ERO que poderiam reagir com ácidos graxos insaturados para formar peróxidos lipídicos. Por outro lado, a glutationa peroxidase destrói os peróxidos de hidrogênio e lipoperóxidos já formados e os converte em água ou em alcoóis relativamente inofensivos. Portanto, Selênio e vitamina E reduzem a taxa de lipoperoxidação e seus danos na membrana celular (Dill \& Rehbun 1985, Moore \& Kohn 1991).

$\mathrm{Na}$ deficiência do sistema antioxidante, especialmente de vitamina E e Se, as ERO lesam as membranas biológicas e provocam a lipoperoxidação, tornando-as defeituosas e permitindo o influxo celular de cálcio. Esse evento promove a ativação das lipases, proteases e endonucleases celular, além do acúmulo de cálcio nas mitocôndrias. As mitocôndrias danificadas não fornecem energia para manter as necessidades energéticas da célula. Quando em excesso, as ERO são mediadores importantes de lesão, tanto induzindo à apoptose quanto à necrose celular (Kumar et al. 2010).

A classificação das miopatias em equinos tende a se expandir rapidamente nos próximos anos, com o melhor reconhecimento das síndromes clínicas e o emprego de técnicas morfológicas, bioquímicas e moleculares no diagnóstico. Desta forma o objetivo desse estudo foi descrever as alterações histológicas e histoquímicas das fibras musculares de equinos acometidos por um surto agudo de miopatia.

\section{MATERIAL E MÉTODOS}

\section{Descrição dos casos}

Sete equinos, da raça Quarta Milha, dois machos e cinco fêmeas, com idade de 18-24 meses foram acometidos. Os animais eram criados em pasto de coast-cross e recebiam suplementação com ração peletizada comercial, feno de alfafa e sal mineral, em um centro de treinamento equestre da região de Botucatu, SP. O surto teve inicio após bruscas alterações climáticas caracterizadas por chuvas fortes e queda de temperatura.

Cinco animais manifestaram uma forma clínica subaguda, com tremores musculares, relutância ao movimento, rigidez muscular e dor a palpação dos músculos glúteos, semitendinoso e semimembranoso, taquipnéia e taquicardia. Os outros dois apresentaram sinais hiperagudos, que além desses incluíam dispnéia, mioglobinúria e decúbito lateral permanente. Todos os animais acometidos apresentaram elevada atividade sérica da creatina kinase (CK), sendo em média de 500UI/dl nos casos subagudos e de 35.000UI/ $\mathrm{dl}$ nos hiperagudos. Desses, um morreu em $72 \mathrm{~h}$ e o outro sobreviveu apesar da intensa atrofia muscular na região lombar, dos membros torácicos e pélvicos. Os demais apresentaram melhora clínica, após terapia com vitamina E e selênio. Nenhum dos eqüinos acometidos apresentou anorexia durante o curso clínico da doença.

\footnotetext{
${ }^{5}$ Hipolador $^{\circledR}$, Rododvia BR 262 Km 123, Borges Sabará, MG 31950-640.

${ }^{6}$ Dixons Surgical Instruments Ltd, Registered in England as No.460740. http://www.dixons-uk.com

${ }^{7}$ Laboratório Arboreto, Rua Paraná 121, Poço Rico, Juiz de Fora, MG 36020-090. Tel. (32) 3212-0385.

${ }^{8}$ Laborana Comércio e Serviços de Aparelhos de Laboratórios Ltda, Rua Diogo Freire 120, Bosque da Saúde, São Paulo, SP 04148-010.
} 


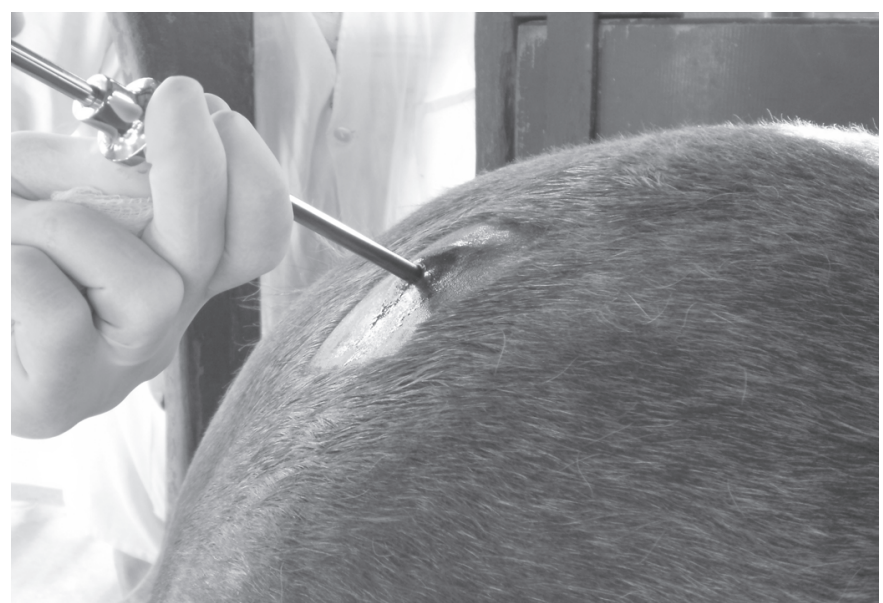

Fig.1. Procedimento de biópsia muscular percutânea, com a agulha tipo Bergström sendo introduzida no músculo glúteo médio de equino.

\section{Biópsia muscular}

Foram realizadas biópsias percutânea do músculo glúteo médio (Fig.1) em todos os equinos acometidos, segundo Padilha et al. (2008). Uma linha imaginária foi traçada da tuberosidade coxal até a crista ilíaca. E numa distância em torno de $20 \mathrm{~cm}$ da tuberosidade coxal foi feita a tricotomia da área na região correspondente ao glúteo médio. Em seguida, foi feito um bloqueio anestésico subcutâneo com, aproximadamente $5 \mathrm{~mL}$ de lidocaína $2 \%^{5}$ sem vasoconstritor.

Após o bloqueio, uma incisão de $1,5 \mathrm{~cm}$ na pele, do tecido subcutâneo e da fáscia muscular superficial foi realizada, utilizando-se

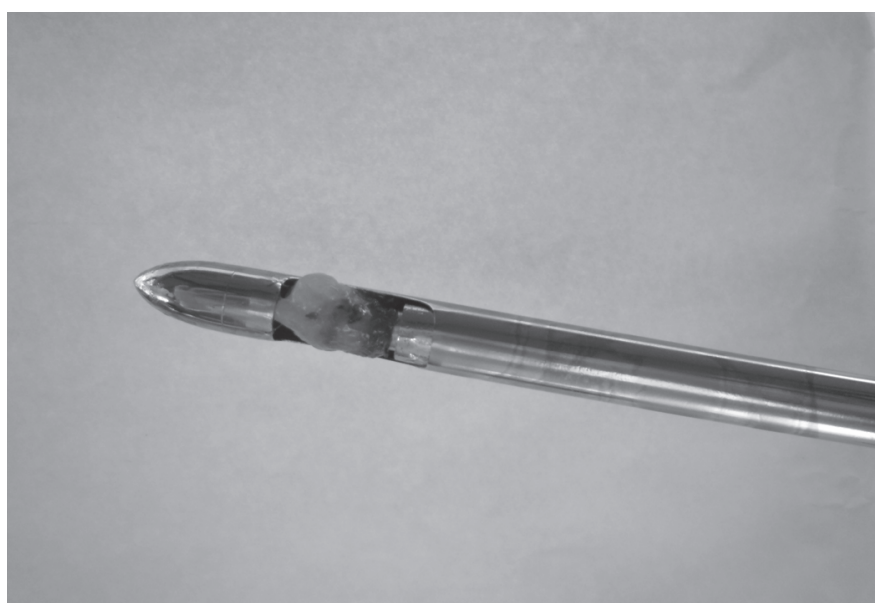

Fig.2. Agulha de Biópsia Muscular tipo Bergström 8.0 com fragmento muscular.

lâmina de bisturi descartável, para que a agulha de biópsia (Fig.2) tipo Bergström $8.0(8 \mathrm{~mm})^{6}$ penetrasse no local. A agulha contendo o cilindro de corte foi inserida através da incisão em direção ao músculo até a profundidade de $60 \mathrm{~mm}$. 0 cilindro foi parcialmente retirado para expor a janela lateral da agulha, que então foi pressionada contra o músculo. Isso permitiu que um pequeno fragmento de músculo fosse introduzido através da janela para o lúmen da agulha. $\mathrm{O}$ fragmento foi cortado, inserindo-se completamente o cilindro de corte. A agulha contendo o fragmento foi retirada e a amostra obtida foi acondicionada por alguns segundos numa placa de Petri fechada com uma gaze embebida em solução salina ${ }^{7}$ para evi-

Fig.3. Corte transversal das fibras musculares esqueléticas provenientes do músculo glúteo médio. 100x. (A) Coloração HE. Nota-se a presença de fibras atróficas e angulares (setas pretas) e fibras vermelhas rajadas (seta vermelha). (B) Coloração pelo Tricrômio de Gomori modificado. Nota-se a presença de fibras vermelhas rajadas (seta vermelha). No detalhe de maior aumento, observam-se depósitos granulares intermiofibrilares e subsarcolemais de coloração vermelha. (C) Reação histoquímica NADH-TR. Fibras do tipo I, IIA e IIX, fibra tipo I atrófica (seta branca), presença de agregados subsarcolemais em fibras tipo I e IIA (setas vermelhas). (D) ATPase pH 9,6. Nota-se a presença de fibras do tipo I vermelhas rajadas (seta vermelha) e fibras tipo II.

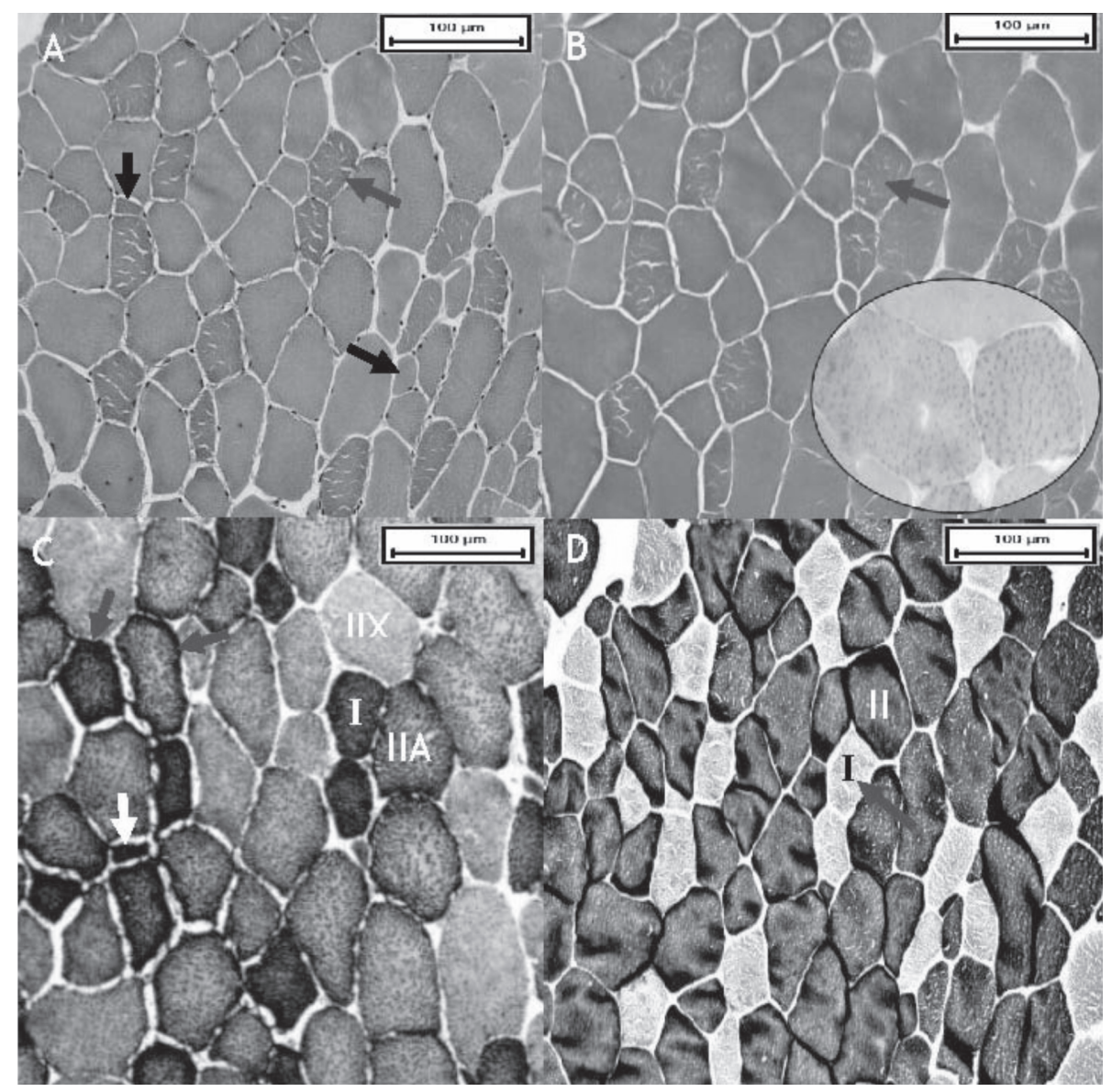




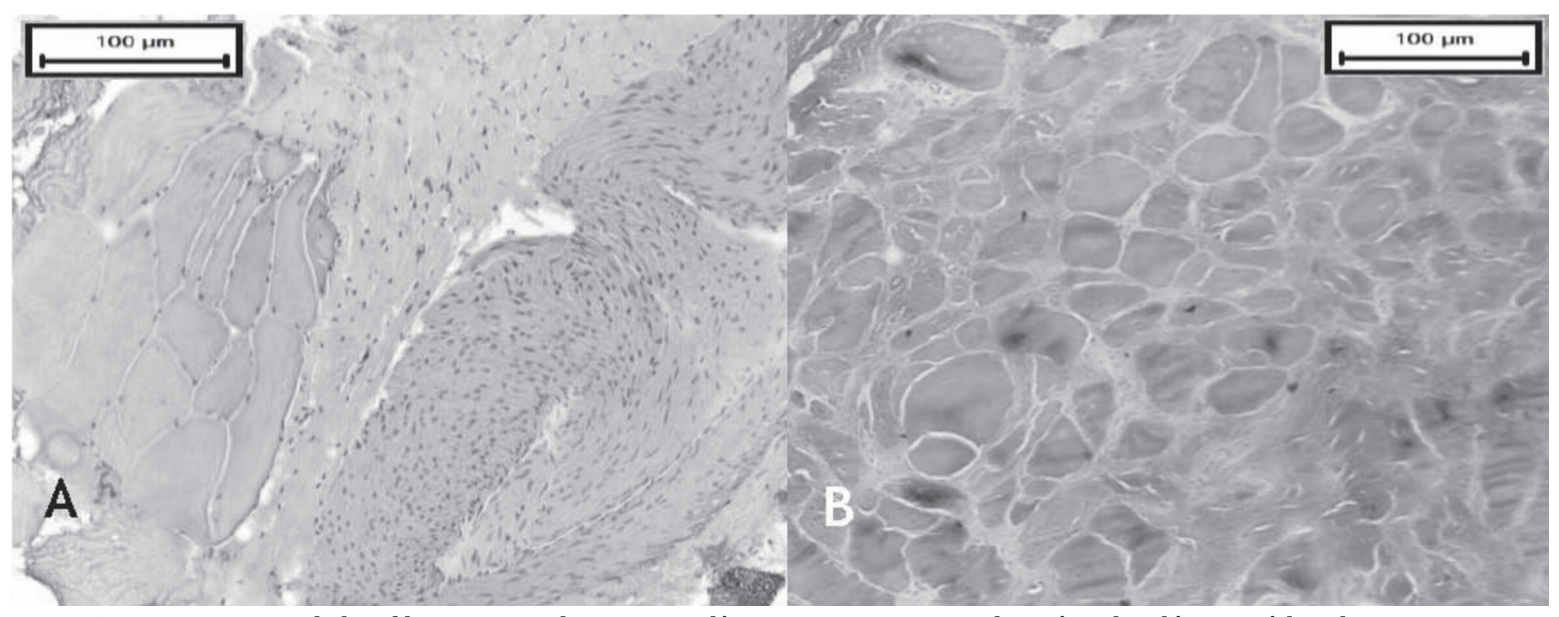

Fig.4. Corte transversal das fibras musculares esqueléticas provenientes do músculo glúteo médio dos animais que apresentaram quadro clínico severo. Nota-se infiltrado mononuclear, fagocitose, intensa proliferação de colágeno, áreas de calcificação e mionecrose. (A) Coloração HE. (B) Coloração pelo Tricômio de Gomory modificado.

tar qualquer atividade contrátil do tecido. Em seguida, o material foi envolto por talco neutro para crioproteção, sendo armazenado em um cassete ${ }^{8}$ para congelação em nitrogênio líquido a uma temperatura de $-96^{\circ} \mathrm{C}$.

\section{Análise histológica e histoquímica do músculo estriado}

Para as análises histológicas e histoquímicas, os fragmentos musculares foram retirados do nitrogênio líquido e colocados em temperatura de $-20^{\circ} \mathrm{C}$ em um criostato para a obtenção de cortes histológicos seriados nos plano transversal com $8 \mu \mathrm{m}$ de espessura.

Para avaliação da arquitetura das fibras musculares foram utilizadas técnicas histológicas de Hematoxilina \& Eosina (HE) e o tricrômico de Gomori Modificado (Dubowitz et al. 2007). Nessas colorações a morfologia das fibras musculares e os elementos não contráteis foram analisados pela microscopia de luz.

As reações histoquímicas realizadas foram a Nicotinamida Adenina Dinucleotídeo-tretrazolium redutase (NADH-TR) para avaliar a capacidade oxidativa das fibras musculares e a ATPase miofibrilar (mATPase, pH 9.6, para classificar os tipos de fibras quanto às características da mATPase (Dubowitz et al. 2007).

As preparações histológicas e histoquímicas foram observadas à microscopia de luz e tiveram os mesmos campos comparados para a análise morfológica e a classificação de três tipos de fibras musculares (I, IIA e IIX). Para tal foram processadas sete amostras musculares, sendo analisadas 200 fibras em cada uma delas.

\section{RESULTADOS}

Nos equinos que apresentaram a forma clínica subaguda $(\mathrm{n}=5)$, a principal alteração morfológica encontrada nas biópsias musculares foi a presença de fibras tipo I e IIA rajadas, observadas principalmente na coloração pelo Tricômio de Gomori modificado (Fig.2B e Fig.3A). Foram observadas também algumas fibras tipo I atróficas e falhas do metabolismo oxidativo com agregados subsarcolemais em fibras tipo I e IIA (Fig.3C,D).

Os achados histopatológicos das biópsias musculares dos animais que apresentaram a forma clínica hiperaguda ( $\mathrm{n}=2$ ) foram: infiltrado inflamatório, aumento do colágeno endo e perimisial, fagocitose, mionecrose e regeneração (Fig.4A,B).

Ao exame necroscópico de um dos animais evidenciou-se áreas pálidas nos músculos glúteo médio, semitendinoso, semimembranoso, longíssimo dorsal, intercostais, diafragma e cardíaco. Os achados histopatológicos dessas áreas incluíam infiltrado mononuclear, fagocitose, intensa proliferação de colágeno e mionecrose segmentar com calcificação.

\section{DISCUSSÃO E CONCLUSÃO}

Segundo Barros (2001), o diagnóstico das miopatias em equinos baseia-se em dados epidemiológicos, nos sinais clínicos associados à patologia clínica e às lesões anatomopatológicas. No diagnóstico diferencial devem ser consideradas as miopatias nutricionais, atípicas, tóxicas, como as causadas por Senna occidentalis e antibióticos ionóforos e miopatias mitocondriais.

A maioria dos surtos agudos de miopatia descrevem uma entidade clínica de início agudo, com caráter sazonal (geralmente no outono), ocorrendo logo após eventos climáticos marcantes (chuvas e frio); há o acometimento de equinos à pasto, não associado ao exercício, com alta mortalidade, presença de mioglobinúria e atividade sérica de creatina kinase elevada (>100.000 UI); presença de degeneração de Zenker e mionecrose predominantemente em fibras do tipo I, em músculos posturais e eventualmente nos músculos respiratórios e cardíacos. Adicionalmente são descritos aspectos histoquímicos e ultraestruturais musculares compatíveis com alterações mitocondriais e lipidose sarcoplasmática, sendo denominada de miopatia atípica ou mioglobinúria atípica (Cassart et al. 2007, Aleman 2008).

Os achados clínico-patológicos dos animais descrito nesse trabalho diferem dos de miopatia atípica pela baixa mortalidade, pelos menores valores de atividade sérica de CK (500UI/dl e 35.000UI/dl nos casos subagudos e hiperagudos, respectivamente) e pela ausência de lipidose sarcoplasmática.

Barros et al. (1999) descrevem que as miopatias tóxicas, como a provocada pela ingestão de Senna occidentalis (fedegoso), causam lesões degenerativas da musculatura estriada cardíaca e esquelética em suínos, bovinos e equinos. Na necropsia observam-se áreas pálidas, focais e/ou coalescentes na musculatura esquelética, além de estrias claras no miocárdio. Além disso, os autores descrevem que histologicamente há vários graus de degeneração, necrose e ruptura de fibras musculares esqueléticas, associadas, algumas vezes, a processos proliferativos e regenerativos. 
Os achados morfológicos das biópsias dos casos subagudos desse estudo diferem dos descritos pelos autores acima, no que diz respeito ao predomínio de lesões nas fibras oxidativas (tipo I e IIA) e à presença de fibras vermelhas rajadas . Essas alterações não foram descritas nos casos de intoxicação por S. occidentalis na literatura consultada (Barros et al. 1999, Vashistha et al. 2009).

Outros achados histopatológicos em eqüinos intoxicados por S. occidentalis são a necrose tubular renal e centrolobular hepática (Vashistha et al. 2009). 0 animal necropsiado nesse estudo não apresentou alterações hepáticas e renais. Além disso, não foi evidenciada a presença de plantas tóxicas, uma vez que o pasto (aproximadamente $10.000 \mathrm{~m}^{2}$ ) e o feno fornecido foram inspecionados.

Bezerra et al. (2000) apontaram a monensina, uma substância produzida pelo fungo Streptomyces cinnamonensis, como um dos antibióticos ionóforos mais usados em medicina veterinária e que os equinos são particularmente sensíveis a essa droga, sendo observado como os principais achados de necrópsia áreas brancas ou amarelas, focais ou focalmente extensas e bilateralmente simétricas nos músculos esqueléticos. Essas lesões foram associadas a edema gelatinoso e translúcido das fáscias intermusculares. Adicionalmente, através de exames histológicos, foi observado miopatia segmentar, multifocal a coalescente. Portanto a intoxicação por ionóforos é um diagnóstico diferencial nos surtos de miopatias em eqüinos. No entanto, os animais desse estudo além de não terem sido expostos à ionóforos, não apresentaram lesões hepáticas e renais, como as descritas por Borges et al. (2001) em casos de intoxicação. Adicionalmente, as lesões musculares provocadas por ionóforos, citadas na literatura, não descrevem o predomínio de lesão em fibras tipo I e IIA e nem a presença de fibras vermelhas rajadas como observadas nos eqüinos acometidos nesse surto.

O maior comprometimento das fibras I e IIA, observado nesse estudo, pode ser justificado em casos de estresse oxidativo decorrente da deficiência dos mecanismos antioxidantes sarcoplasmáticos. Segundo Stashak (2006) e Aleman (2008), as alterações musculares observadas na miopatia nutricional pelas reações histoquímicas são mais evidentes nas fibras tipo I e IIA, pois estão diretamente relacionadas ao estresse oxidativo decorrente do desequilíbrio do sistema antioxidante, por conta da deficiência de vitamina E e Se. De acordo com Snow \& Valberg (1994) as fibras do tipo I apresentam maior irrigação sanguínea, utilizam metabolismo oxidativo como fonte de energia, são mais resistentes à fadiga, têm maior quantidade de mitocôndria, coram-se fortemente à NADH-TR e fraco à ATPase 9,6. Já as fibras do tipo II apresentam metabolismo glicolítico como fonte de energia, são menos resistentes à fadiga, têm menor quantidade de mitocôndrias, coram-se forte à ATPase 9,6 e fraco à NADH-TR.

De acordo com Radostits et al. (2002) e Kirschvink et al. (2008), quando há prejuízo dos mecanismos anti-oxidantes, como na deficiência de vitamina $\mathrm{E}$ e Se, as membranas celulares tornam-se defeituosas, permitindo o influxo de cálcio para o citosol, ativando lipases, proteases, endonucleases e danificando as mitocôndrias. A partir dessa alteração, as mitocôndrias lesadas tornam-se incapazes de evitar o estresse oxidativo e de fornecer energia para a homeostase celular, o que pode inicialmente provocar o aparecimento das fibras vermelhas rajadas, e com a evolução da doença resultar em morte celular.

Segundo Yoshida (1997), Dubowitz \& Sewry (2007) e Graça et al. (2008), através da coloração do Tricômio do Gomori modificado pode-se observar a presença das fibras vermelhas rajadas. Essas fibras são descritas como agregados mitocondriais intermiofibrilares que aparecem como depósitos granulares de coloração vermelha e com uma espessa camada subsarcolemal. Além disso, Kumar et al. (2010), descreveram que esses agregados são ricos em enzimas oxidativas e que estão ligadas às alterações do metabolismo oxidativo e das funções mitocondriais como ocorrem nas miopatias mitocondriais. Esses achados são semelhantes aos observados nesse estudo, no qual a presença das fibras vermelhas rajadas podem estar relacionadas com a ocorrência da miopatia nutricional subaguda em eqüinos.

Dubowitz \& Sewry (2007) e Graça et al. (2008) descrevem a ocorrência de fibras vermelhas rajadas associadas a miopatias mitocondriais em seres humanos. Valberg et al. (1994) descreveram um caso de miopatia mitocondrial por deficiência do complexo enzimático I da cadeia respiratória, em uma égua com histórico de intolerância ao exercício e eventuais episódios de rigidez muscular. A histoquímica muscular revelou anormalidades nas mitocôndrias caracterizadas pela intensa reação e presença de agregados subsarcolemais na coloração de NADH-TR e tricrômio de Gomori. Porém, poucas fibras apresentaram aparência rajada, como descrito nas miopatias mitocondriais humanas.

A forma esquelética da miopatia nutricional, como descrito por Radostits et al. (2002), Smith (2006) e Aleman (2008), apresenta início insidioso, não associada ao exercício, caracterizado por fraqueza e/ou rigidez, tremores e dor à palpação muscular, relutância em se mover, taquicardia, arritmias, taquipnéia, elevada atividade sérica da CK, mioglobinúria, decúbito lateral permanente e morte. Os animais desse estudo apresentaram sinais clínicos compatíveis com a miopatia nutricional como: tremores musculares, relutância ao movimento, rigidez e dor a palpação muscular, taquipnéia, taquicardia, mioglobinúria e decúbito lateral permanente. Todos os animais acometidos apresentaram elevada atividade sérica da CK, variando de $500 \mathrm{UI} / \mathrm{dl}$ a $35.000 \mathrm{UI} / \mathrm{dl}$.

Os sete eqüinos desse estudo sofreram de uma miopatia de início agudo e progressiva evolução clínica. Desse modo, as características descritas coincidem com os achados de Cassart et al. (2007), onde ocorreu o acometimento repentino de miopatia atípica em vários animais ao mesmo tempo e de Radostits et al. (2002), Smith (2006) e Aleman (2008), que também descreveram relatos de miopatia nutricional.

Quanto à idade dos animais, os casos relatados no presente estudo estão de acordo com os estudos de Cassart et al. (2007) e Aleman (2008) sobre miopatia atípica e miopatia nutricional, que descreveram a maior incidência em animais jovens. De acordo com Koller et al. (1984) e Campbell et al. (1990), a miopatia nutricional acomete todos os animais pecuários, sendo detectada mais comumente em animais jovens e de rápido crescimento, como os eqüinos de 18-24 meses de idade descritos nesse surto.

Outros aspectos epidemiológicos observados nesse estu- 
do são comuns as descrições de miopatia atípica e miopatia nutricional, como a prévia ocorrência de eventos climáticos marcantes provocando estresse e a criação dos animais à pasto sem ou com pouca suplementação alimentar, conforme descrito por Radostits et al. (2002). Esse último aspecto pode predispor ao aparecimento da miopatia nutricional.

De forma semelhante, Cassart et al. (2007) e Aleman (2008) observaram que, na miopatia atípica, o surgimento dos sinais clínicos também não está relacionado com atividade física e incluem sinais semelhantes como fraqueza, tremores musculares, relutância em se locomover e andar rígido, dor a palpação muscular, taquicardia, taquipnéia, dispnéia, elevação da atividade sérica de $\mathrm{CK}$, mioglobinúria e alta mortalidade. Portanto, os animais desse estudo apresentaram manifestações clínicas compatíveis tanto com a miopatia nutricional quanto com a miopatia atípica, não sendo possível diferenciar clinicamente essas duas enfermidades, fato esse que está de acordo com os pressupostos de Valberg et al. (1999) e Votion et al. (2006).

Por outro lado, os casos de miopatia atípica descritos por Hosie et al. (1986) e Votion \& Serteyn (2008) não responderam a terapia com vitamina $\mathrm{E}$ e Se, o que difere do observado nesse surto onde houve melhora clínica dos animais que não apresentaram decúbito permanente. Já os dois casos que apresentaram decúbito permanente, a terapia não foi eficiente, pois um animal morreu e o outro, apesar de ter sobrevivido, desenvolveu atrofia muscular severa.

Baseado em Maas (1994), Parish \& Hodopson (1994) e Radostits et al. (2002), as análises histológicas e histoquímicas do tecido muscular acometido demonstram necrose segmentar com calcificação de segmentos necróticos das fibras musculares. Adicionalmente, Lofstedt (1997) e Perkins et al. (1998) relataram que à necrópsia observa-se acometimento bilateral da musculatura esquelética com áreas de degeneração de aspecto esbranquiçado ou acinzentado e o músculo apresentava-se pálido com estrias brancas, representando necrose coagulativa e edema. Comumente, os músculos afetados incluem o miocárdio, músculo torácico, intercostal, pélvico e cervical, diafragma, faringe e músculos mastigatórios. Lofstedt (1997) ainda descreveu que as características histológicas incluem infiltrado, mionecrose, fibrose e calcificação nos casos crônicos. Adicionalmente, tanto na miopatia nutricional quanto na miopatia atípica podem ser observadas áreas pálidas nos músculos posturais, respiratórios e miocárdio, segundo estudos de Votion et al. (2006). Esses achados são compatíveis com características observadas no exame post mortem de um dos animais desse estudo.

Na histologia dos músculos esqueléticos foi observada necrose e calcificação, sendo semelhantes aos achados descritos na literatura por Hulland (1993), Jones et al. (1997) e Pugh (2002), onde foi citado que a calcificação é um achado comum na miopatia nutricional.

A aparente resposta à terapia maciça com vitamina $\mathrm{E} e \mathrm{Se}$ e a baixa mortalidade quando comparado aos relatos de miopatia atípica, indicam que esse surto foi provocado por deficiência de vitamina E e Se, o qual desencadeou lesões mitocondriais caracterizadas pelas fibras musculares vermelhas rajadas, sendo compatível com o diagnóstico de miopatia nutricional.
Diante dos achados morfológicos desse estudo conclui-se que a biópsia muscular tem um importante papel no diagnóstico das miopatias, sendo que na miopatia nutricional as alterações da fase inicial da doença ou dos casos subagudos podem ser observadas pelo comprometimento predominante de fibras tipo I e IIA e pela presença de fibras vermelhas rajadas. Já nos casos crônicos, ou mais severos, observa-se a presença de algumas fibras atróficas, outras hipertróficas, proliferação de colágeno, segmentos necróticos e áreas de calcificação.

\section{REFERÊNCIAS}

Aleman M. 2008. A review of equine muscle disorders. Neuromuscular Disorders 18:277-287.

Barros C.S.L. 2001. Deficiência de selênio e vitamina E, p.329-333. In: RietCorrea F., Schild A.L., Méndez M.C. \& Lemos R.A.A. (Eds), Doenças de Ruminantes e Eqüinos. Varela, São Paulo,

Barros C.S.L., Ilha M.R.S., Bezerra P.S., Langohr I.M. \& Kommers G.D. 1999. Intoxicação por Senna occidentalis (Leg. Caesalpinoideae) em bovinos em pastoreio. Pesq. Vet. Bras. 19(2):68-70.

Bezerra Jr P.S., Ilha M.R.S., Langohr I.M. \& Barros C.S.L. 2000. Intoxicação experimental por monensina em eqüinos. Pesq. Vet. Bras. 20(3):102-108.

Borges A.S., Silva D.P.G., Gonçalves R.C., Kuchembuck M.R.G., Chiacchio S.B., Mendes L.C.N. \& Bandarra E.P. 2001. Ionóforos e equinos: uma combinação fatal. Revta Educ. Contin. CRMV-SP 4(2):33-40.

Burk R.F. \& Hill K.E. 1993. Regulation of selenoproteins. Annu. Rev. Nutr. 13:65.

Campbell D.T., Maas J., Weber D.W., Hedstrom O.R. \& Norman B.B. 1990. Safety and efficacy of two sustained-release intrareticular selenium supplements and the associated placental and colostral transfer of selenium in beef cattle. Am. J. Vet. Res. 51(5):813-817.

Cassart D., Baise E., Cherel Y., Delguste C., Antoine N., Votion D., Amory H., Rollin F., Linden A., Coignoul F. \& Desmecht D. 2007. Morphological alterations in oxidative muscles and mitochondrial structure associated with equine atypical myopathy. Equine Vet. J. 39:26-32.

Combs G.F. \& Combs S.B. 1986. The role of selenium in nutrition. Academic Press, London. 180p.

Dill S.G. \& Rehbun W.C. 1985. White muscle disease in foals. Compend. Cont. Educ. Pract. Vet. 7:627-636.

Dubowitz V. \& Sewry C.A. 2007. Histological and Histochemical stains and reactions, p.21-39. In: Ibid. (Eds), Muscle Biopsy: A practical approach. $3^{\text {rd }}$ ed. W.B. Saunders, Philadelphia.

Graça C.R., Kouyoumdjian J.A. \& Marie S.K.N. 2008. Biópsia muscular com estudo histoquímico: experiência iniciada na Faculdade de Medicina de São José do Rio Preto. Arq. Ciênc. Saúde 15(1):24-28.

Higuchi T., Ichijo S., Osame S. \& Ohishi H. 1989. Studies on serum selenium and tocopherol in white muscle disease of foal. Nippon Juigaku Zasshi 51:52-59.

Hosie B.D., Gould P.W., Hunter A.R., Low J.C., Munro R. \& Wilson H.C. 1986. Acute myopathy in horses at grass in East and South-East Scotland. Vet. Rec. 119:444-449.

Hulland T.J. 1993. Muscle and tendon, p.230-234. In: Jubb K.V.F., Kennedy P.C. \& Palmer N. (Eds), Pathology of Domestic Animals. Vol.1. $4^{\text {th }}$ ed. Academic Press, San Diego.

Jones T.C., Hunt R.D. \& King N.W. 1997. Patologia Veterinária. 6ª ed. Manole, São Paulo, p.830-850.

Kirschvink N., De Moffarts B. \& Lekeux P. 2008. The oxidant/antioxidant equilibrium in horses. Vet. Journal 177(2):178-191.

Koller L.D., Whitbeck G.A. \& South P.J. 1984. Transplacental transfer and colostral concentrations of selenium in beef cattle. Am. J. Vet. Res. 45(12): 2507-2510.

Kumar V., Abbas A.K., Fausto N. \& Aster J.A. 2010. Robbins and Cotran: Pathologic basis of disease. $8^{\text {th }}$ ed. W.B. Saunders, Philadelphia. $1450 \mathrm{p}$. 
Lewis S.S., Valberg S.J. \& Nielsen I.L. 2007. Suspected immune-mediated myositis in horses. J. Vet. Intern. Med. 21:495-503.

Lofstedt J. 1997. White muscle disease of foals. Vet. Clin. North Am., Equine Pract. 13:169-185.

Maas J. 1994. Miodegeneração nutricional, p.1335-1351. In: Smith B.P. (Ed.), Tratado de Medicina Interna de Grandes Animais. Manole, São Paulo.

Martin W.M.C., McNally N.J. \& De Ronde J. 1981. Enhancement of the effect of cytotoxic drugs by radiosensitizers. Brit. J. Cancer 43:756.

Moore R.M. \& Kohn C.W. 1991. Nutritional muscular dystrophy in foals. Compend. Contin. Educ. Pract. Vet. 13:476-489.

Padilha F.G.F., Suma R. \& Amorim R.M. 2008. Avaliação prática da biópsia muscular percutânea por agulha em equinos. Hora Vet. 28:29-32.

Parish S.M. \& Hodopson D.R. 1994. Miodegeneração nutricional, p.13351351. In: Smith B.P. (Ed.), Tratado de Medicina Interna de Grandes Animais. Manole, São Paulo.

Peek S.J., Semrad S.D. \& Perkins G.A. 2003 Clostridial myonecrosis in horses (37 cases 1985-2000). Equine Vet. J. 35:86-92.

Perkins G., Valberg S.J., Madigan J.M., Carlson G.P. \& Jones S.L. 1998. Electrolyte disturbances in foals with severe rhabdomyolysis. J. Vet. Intern. Med.12:173-177.

Pugh D.G. 2002. Sheep and Goat Medicine. W.B. Saunders, Philadelphia, p.223-255.

Radostits E.M., Gay C.C., Blood D.C. \& Hinchciff K.W. 2002. Clínica Veterinária. 9ª ed. Guanabara Koogan, São Paulo, p.1364-1384.

Smith B.P. 2006. Medicina Interna de Grandes Animais. $3^{a}$ ed. Manole, Barueri, SP, Cap.40, p.1279.

Snow D.H. \& Valberg S.J. 1994. Muscle anatomy, physiology, and adaptation to exercise and training, p.170. In: Hodgson D.R. \& Rose R.J. (Eds), The Athletic Horse. W.B. Saunders, Philadelphia.
Sponseller B.T., Valberg S.J., Tennent-Brown B.S., Foreman J.H., Kumar P. \& Timoney J.F. 2005. Severe acute rhabdomyolysis associated with Streptococcus equi infection in four horses. J. Am. Vet. Med. Assoc. 227:18001807.

Stashak T.S. 2006. Claudicação em Eqüinos segundo Adams. 5ª ed. Editora Roca, São Paulo.

Valberg S., Cardinet III G.H., Carlson G.P. \& DiMauro S. 1992. Polysaccharide storage myopathy associated with exertional rhabdomyolysis in the horse. Neuromuscular Disorders 2:351-359.

Valberg S.J., Carlson G.P., Cardinet G.H., III Birks E.K., Jones J.H., Chomyn A. \& DiMauro S. 1994. Skeletal muscle mitochondrial myopathy as a cause of exercise intolerance in a horse. Muscle and Nerve 17:305-312.

Valberg S.J., Michelson J.R., Galland E.M., MacLeay J.M., Lentz L. \& De La Corte F. 1999. Exertional rhabdomyolysis in quarter horses and thorough-breds: One syndrome, multiple aetiologies. Equine Vet. J. (Suppl30):533-538.

Vashishtha V.M., John T.J. \& Kumar A. 2009. Clinical and pathological features of acute toxicity due to Cassia occidentalis in vertebrates. Indian J. Med. Res. 130:23-30.

Votion D.M., Amory H., Demoulin V., Desmecht D., Rollin F., Thiry E. \& Baise E. 2006. Atypical myopathy (Atypical myoglobinuria). Disponível em <http://www.ivis.org/reviews/rev/votion/chapter.asp?LA=1> Acesso 12 Jul. 2010.

Votion D.M. \& Serteyn D. 2008. Equine atypical myopathy: A review. Vet. Journal 178:185-190.

Ward J.J., Sodhi J.S., McGufn L.J., Buxton B.F. \& Jones D.T. 2004. Prediction and functional analysis of native disorder in proteins from the three kingdoms of life. J. Mol. Biol. 337:635-645.

Yoshida K. 1997. Morphological study of muscle fibers stained red by modified Gomori trichrome staining with special reference to smooth red fibers. Hokkaido Igaku Zasshi 72(2):163-180. 